\title{
A checklist of Rutelinae MacLeay, 1819 (Coleoptera, Melolonthidae) of Bahia, Brazil
}

\author{
André da Silva Ferreira ${ }^{1 *}$ (D), Lúcia M. Almeida ${ }^{2}$ Freddy Bravo ${ }^{3} \&$ Paschoal Coelho Grossi $^{4}$ \\ ${ }^{1}$ Universidade Federal da Bahia, Instituto de Biologia, Programa de Pós-Graduação em Diversidade Animal, 40170-115, \\ Salvador, BA, Brasil \\ ${ }^{2}$ Universidade Federal do Paraná, Departamento de Zoologia, Laboratório de Sistemática e Bioecologia de \\ Coleoptera, 81531-980, Curitiba, PR, Brasil \\ ${ }^{3}$ Universidade Estadual de Feira de Santana, Departamento de Ciências Biológicas, Laboratório de Sistemática de \\ Insetos, 44036-900, Feira de Santana, BA Brasil \\ ${ }^{4}$ Universidade Federal Rural de Pernambuco, Rua Dom Manoel de Medeiros, 52171-900, Recife, PE, Brasil \\ *Corresponding author: André da Silva Ferreira, e-mail: sferreira.and@gmail.com
}

FERREIRA, A. S., ALMEIDA, L. M., BRAVO, F., GROSSI, P. C. A checklist of Rutelinae MacLeay, 1819 (Coleoptera, Melolonthidae) of Bahia, Brazil. Biota Neotropica. 18(2): e20170476. http://dx.doi.org/10.1590/1676-0611-BN-2017-0476

\begin{abstract}
A list of species of Rutelinae from Bahia state, Northeastern Brazil, is presented. The list is based on specimens deposited in Brazilian collections. The list includes 4 tribes, 23 genera, 101 species and 17 subspecies. The genera Byrsopolis Burmeister, 1844, Pseudodorysthetus Soula, 2008 and Trizogeniates Ohaus, 1917 are recorded for the first time in Bahia and Northeastern Brazil. Thirty species are newly recorded in Bahia: Areoda espiritosantensis Ohaus, 1905, B. laticollis Burmeister, 1855, Bolax flavolineata (Mannerheim, 1829), Chlorota abdominalis Ohaus, 1926, C. espiritosantensis Ohaus, 1912, Dorysthetus espiritosantensis Ohaus, 1905, D. fulgidus (Waterhouse, 1881), Leucothyreus acanthurus Ohaus, 1917, L. albopilosus Ohaus, 1917, L. campestris Burmeister, 1855, L. cayapo Ohaus, 1931, L. duplopunctatus Frey, 1976, L. eligius Ohaus, 1918, L. fluminensis Ohaus, 1918, L. iridipennis Ohaus, 1917, L. lucipetens Ohaus, 1931, L. occipitalis Ohaus, 1931, L. pallefactus Ohaus, 1924, L. paulista Ohaus, 1917, L. punctulatus Blanchard, 1851, L. suturalis Laporte, 1840, L. trochantericus Ohaus, 1917, L. verticalis Ohaus, 1924, Macraspis cincta (Drury, 1872), Paranomala tricostulata (Ohaus, 1897), P. violacea (Burmeister, 1844), Pseudodorysthetus calcaratus (Spinola, 1835), and Trizogeniates planipennis Ohaus, 1917. Pelidnota unicolor unicolor (Drury, 1778) is recorded for the first time in Bahia. Fourteen species are identified and will be described in subsequent papers: 10 of Leucothyreus MacLeay, 1819, 2 of Lobogeniates Ohaus, 1917 and 1 species of Byrsopolis Burmeister, 1844 and Pelidnota MacLeay, 1819. Rutelini is the richest tribe with 16 genera and 49 species. The information presented in the list generates an important set of knowledge regarding the diversity of Rutelinae of Bahia and Brazil and provides the basis for conducting future research within the group.
\end{abstract}

Keywords: new records, Northeastern Brazil, species list.

\section{Um checklist dos Rutelinae MacLeay, 1819 (Coleoptera, Melolonthidae) da Bahia, Brasil}

Resumo: É apresentada uma lista de espécies de Rutelinae do estado da Bahia, Nordeste do Brasil. A lista é baseada em espécimes depositados em coleções brasileiras. A lista inclui quatro tribos, 23 gêneros, 101 espécies e 17 subespécies. É feito o primeiro registro para a Bahia e região Nordeste dos gêneros Byrsopolis Burmeister, 1844, Pseudodorysthetus Soula, 2008 e Trizogeniates Ohaus, 1917. Trinta espécies de Rutelinae foram registradas pela primeira vez para a Bahia e região Nordeste: Areoda espiritosantensis Ohaus, 1905, B. laticollis Burmeister, 1855, Bolax flavolineata (Mannerheim, 1829), Chlorota abdominalis Ohaus, 1926, C. espiritosantensis Ohaus, 1912, Dorysthetus espiritosantensis Ohaus, 1905, D. fulgidus (Waterhouse, 1881), Leucothyreus acanthurus Ohaus, 1917, L. albopilosus Ohaus, 1917, L. campestris Burmeister, 1855, L. cayapo Ohaus, 1931, L. duplopunctatus Frey, 1976, L. eligius Ohaus, 1918, L. fluminensis Ohaus, 1918, L. iridipennis Ohaus, 1917, L. lucipetens Ohaus, 1931, L. occipitalis Ohaus, 1931, L. pallefactus Ohaus, 1924, L. paulista Ohaus, 1917, L. punctulatus Blanchard, 1851, L. suturalis Laporte, 1840, L. trochantericus Ohaus, $1917, L$. verticalis Ohaus, 1924, Macraspis cincta (Drury, 1872), Paranomala tricostulata (Ohaus, 1897), P. violacea (Burmeister, 1844), Pseudodorysthetus calcaratus (Spinola, 1835) e Trizogeniates planipennis Ohaus, 1917. Foi feito o primeiro registro de Pelidnota unicolor unicolor (Drury, 1778) para a Bahia. Foram identificadas 14 espécies, que serão descritas em trabalhos posteriores: dez de Leucothyreus MacLeay, 1819, duas de Lobogeniates Ohaus, 1917 e uma de Byrsopolis Burmeister, 1844 e Pelidnota MacLeay, 1819. A tribo Rutelini apresenta a maior riqueza com 16 gêneros e 49 espécies. Os resultados aqui apresentados têm importantes informações para o conhecimento de Rutelinae, no estado da Bahia e no Brasil, e servirão de base para a realização de futuras pesquisas com o grupo.

Palavras-chave: lista de espécies, novos registros, Região Nordeste do Brasil. 


\section{Introduction}

According to Kohlmann \& Morón (2003) Scarabaeoidea is represented by three families: Lucanidae, Passalidae and Scarabaeidae, with this last family divided into two informal categories, "Laparosticti" (with spiracles positioned on the pleural membrane) and "Pleurosticti" (with spiracles positioned on the superior part of the abdominal ventrite). The scarabaeids include two feeding groups: coprophagous consisting of subfamilies Aphodiinae and Scarabaeinae ("Laparosticti") and the phytophagous consisting of subfamilies Cetoniinae, Dynastinae, Melolonthinae and Rutelinae ("Pleurosticti") (Lawrence \& Newton 1995). Cherman \& Morón (2014) recently considered the six phytophagous subfamilies in Melolonthidae (Euchirinae, Dynastinae, Hopliinae, Melolonthinae, Rutelinae and Sericinae). This paper follows the classification of Cherman \& Morón (2014)

Rutelinae is the second largest subfamily of Melolonthidae according to the number of species, with approximately 4,197 species described in the world but with the greatest richness in the tropics (Hardy 1991, Jameson 2002, Ratcliffe \& Jameson 2005, Krajcik 2008, Jameson \& Ratcliffe 2011, Morón \& Ramírez-Ponce 2012). Melolonthinae is the richest subfamily of Melolonthidae, with about 11,000 known species (Evans 2002). Seven tribes are currently recognized in Rutelinae: Adoretini, Alvarengiini, Anatistini, Anomalini, Anoplognathini, Geniatini and Rutelini (Bouchard et al. 2011).

Adult ruteline are phytophagous, playing an important ecological role in pollination of some plant species. Larvae are saprophytophagus and contribute directly to the decomposition of dead organic matter deposited in forests and in the nutrient cycle (Hardy 1991, Morón et al., 1997, Paucar-Cabrera 2003), while some species feed on roots and sometimes become pests (Ritcher 1958, Jameson et al. 2003, Jameson \& Howkins 2005). In Brazil, Paranomala testaceipennis (Blanchard, 1851) was recorded in agricultural crops and pasture areas in Mato Grosso do Sul (Rodriguez-del-Bosque 1996, 1998, Rodrigues et al. 2008); Leucothyreus albopilosus Ohaus, 1917 was recorded on Eucalyptus citriodora (Hook, 1848); L. dorsalis Blanchard, 1850 was associated to the roots of Acrocomia aculeata (Jacq) Lodd. ex Mart. (Arecaceae) from Mato Grosso do Sul (Puker et al. 2009, Pereira et al. 2013).

In the Neotropical Region, approximately 1,337 species of Rutelinae were recorded (Morón 1990, 2004, Jameson 2008, Soula 2011, Filippini et al. 2016, Ferreira et al. 2017, Howkins 2017, Moore et al., 2017, Seidel et al. 2017, Sierra 2017), while 436 are from Brazil (Grossi \& Vaz-de-Mello 2016, Ferreira et al. 2017). Grossi \& Vaz-de-Mello (2016) also included 104 subspecies and 58 genera in their list from Brazil. Only one inventory of Rutelinae is known from Bahia State (Viana et al. 2001), which was performed with light traps in Cruz das Almas municipality (approximately $\left.12^{\circ} 40^{\prime} \mathrm{S}-39^{\circ} 06^{\prime} \mathrm{W}\right)$. The authors recorded species of four genera: Paranomala Casey, 1915 (Anomalini), Geniates Kirby, 1819 Leucothyreus MacLeay, 1819 (Geniatini) and Pelidnota MacLeay (Rutelini), however, only one species was named and identified, Pelidnota fulva Blanchard, which is very probably a misidentified species, as this taxon occurs in another geographical region, according to the last revision (Soula 2009).

The most recent information on the diversity of the species from Bahia is scattered among 17 papers (Jameson 1996, Jameson \& Hawkins 2005, Jameson \& Ratcliffe 2011, Krajcik 2008, Machatschke 1972, Soula 2002a, b, 2003, 2005, 2006, 2008, 2009, 2010, 2011, Ratcliffe \& Jameson 1989, Viana et al. 2001, Ferreira et al. 2017) and includes 61 species and nine subspecies in 20 genera of the tribes Anatistini, Anomalini, Geniatini and Rutelini.

In this paper, we present an updated species list of Rutelinae from Bahia that are deposited in seven collections in South, Southeast and Northeast of Brazil. Information about the geographic distribution of the species listed is also provided. The data presented here gather important information that adds new knowledge about the diversity of Rutelinae in
Brazil and the Neotropical region and serves as a basis for conducting future research with the group.

\section{Material and Methods}

Bahia is one of the biggest states of Brazil (Figure 1) with an area of $564.733,177 \mathrm{~km}^{2}$ (Instituto Brasileiro de Geografia e Estatística-IBGE 2013), which represents $37.7 \%$ of all the Northeastern territory (Bahia 2013). The vegetation, climate, and altitudinal range are heterogeneous, with the presence of three Brazilian Biomes (Caatinga, Atlantic Forest and Cerrado), ecosystems of restingas, mangroves, campos rupestres, and ecotones between the biomes, rainforests, semi-deciduous forest and high montane vegetation, among others (Bahia 2013).

The collections visited for this study were (acronyms according to Evenhuis (2009) have been used when available): CEIOC - Entomological Collection of Instituto Oswaldo Cruz, Rio de Janeiro, Brazil; CERPE - Entomological Collection of Universidade Federal Rural de Pernambuco, Recife, Pernambuco, Brazil; DZUP - Entomological Collection Pe. J. S. Moure, Department of Zoology, Universidade Federal do Paraná, Curitiba, Paraná, Brazil; EPGC - Everardo and Paschoal Grossi Collection, Nova Friburgo, Rio de Janeiro, Brazil; MNRJ - National Museum, Universidade Federal do Rio de Janeiro, Rio de Janeiro, Brazil; MZFS - Collection Prof. Johann Becker, Museum of Zoology of Universidade Estadual de Feira de Santana, Bahia, Brazil; and MHNBA/MZUFBA - Entomological Collection of Museum of Natural History/Zoology of Bahia State/Universidade Federal da Bahia, Salvador, Bahia, Brazil.

The species identification was based on Frey (1976), Ohaus (1905, 1912, 1913, 1917, 1918a, 1918b, 1924, 1926, 1928, 1930, 1931), Soula $(1998,2006,2009,2010,2011)$, and by comparison with the studied collections.

For the study of male genitalia, each specimen was immersed in boiling water for approximately two to four minutes and the genitalia removed, then studied under a stereomicroscope, glued in a triangle, and pinned just below the specimen.

The geographical coordinates, when not available on the label of the specimen, were obtained by the geoLoc tool on the speciesLink online data platform (http://splink.cria.org.br/geoloc), using the IBGE as a source for the data recovery. The geographical records were plotted on the map of Bahia using QGIS version 2.10.1. The final artwork for the map of geographical records and richness of genera and species by tribe was executed using Abobe Photoshop CS6 ${ }^{\circledR}$.

The information transcribed from the labels of the examined material usually adhered to the following pattern: COUNTRY, State: ("Locality"; "additional information"); (Geographic Coordinates), Data, Collector. (ACRONYM OF THE MUSEUM\# register number).

\section{Results and Discussion}

A total of 1,495 specimens, of 20 genera, 79 species and 12 subspecies of the tribes Anomalini, Anatistini, Geniatini and Rutelini were examined. Before the current study, 23 genera, 101 species and 17 subspecies of Rutelinae from Bahia (Table 1) had been reported. With this study, 32 species, 11 subspecies and the genera Anticheiroides Soula, 1998, Oplognathus MacLeay, 1819 and Parhoplognathus Ohaus, 1930, cited from Bahia in the literature by Machatschke (1972), Soula (1998, 2008), and Moore et al. (2017) respectively were not found in the collections studied (Table 1).

The genera Byrsopolis Burmeister, 1844, Pseudodorysthetus Soula, 2008 and Trizogeniates Ohaus, 1917 were first recorded for Bahia and the Northeast region. Thirty species of Rutelinae were recorded for the first time in Bahia and Northeastern Brazil: Areoda espiritosantensis Ohaus, 1905, Byrsopolis aff. castanea Burmeister, 1844, Bolax flavolineata (Mannerheim, 1829), Chlorota abdominalis Ohaus, 1926, C. espiritosantensis Ohaus, 
Table 1. List of species and subspecies of Rutelinae from Bahia state, Northeast - Brazil adding the literature data with the results presented in this study and, geographic distribution.

\begin{tabular}{|c|c|}
\hline SPECIES/SUBSPECIES & DISTRIBUTION \\
\hline \multicolumn{2}{|l|}{$\begin{array}{l}\text { ANOMALINI Streubel, } 1839 \\
\text { Anomalina Streubel, } 1839 \\
\text { Paranomala Casey, } 1915\end{array}$} \\
\hline Paranomala chromicolor (Burmeister, 1855) & Brazil (Bahia and Pará) (Machatschke 1972, Grossi \& Vaz-de-Mello 2016) \\
\hline Paranomala foveiceps (Ohaus, 1897) & Brazil (Bahia) (Machatschke 1972, Krajcik 2008, Grossi \& Vaz-de-Mello 2016) \\
\hline Paranomala inconstans (Burmeister, 1844) & $\begin{array}{l}\text { Mexico, Central and South America, in Brazil (Bahia and Rio de Janeiro) (Machatschke 1972, Krajcik } \\
\text { 2008, Grossi \& Vaz-de-Mello } 2016 \text { and present study) }\end{array}$ \\
\hline Paranomala tricostulata (Ohaus, 1897) & Brazil (Amazonas, Bahia (new occurrence register)) and Colombia (Mozo) (Ohaus 1897) \\
\hline Paranomala undulata undulata (Melsheimer, 1844) & $\begin{array}{l}\text { North, Central and South America (Machatschke 1972, Krajcik 2008, Grossi \& Vaz-de-Mello } 2016 \text { and } \\
\text { present study) }\end{array}$ \\
\hline Paranomala undulata varians (Burmeister, 1844) & North America (Mexico), Brazil (Machatschke 1972, Krajcik 2008 and present study) \\
\hline Paranomala violacea (Burmeister, 1844) & $\begin{array}{l}\text { Brazil (Bahia (new occurrence register), Espírito Santo, Santa Catarina) (Machatschke 1972, Krajcik } \\
\text { 2008, Grossi \& Vaz-de-Mello 2016) }\end{array}$ \\
\hline \multicolumn{2}{|l|}{$\begin{array}{l}\text { RUTELINI MacLeay, } 1819 \\
\text { Anticheirina Lacordaire, } 1856 \\
\text { Anticheiroides Soula, } 1998\end{array}$} \\
\hline Anticheiroides inauratus bahianus Soula, 1998 & Brazil (Bahia) (Soula 1998) \\
\hline \multicolumn{2}{|l|}{ Chlorota Burmeister, 1844} \\
\hline Chlorota abdominalis Ohaus, 1926 & Brazil (Amazonas, Bahia (new occurrence register)) (Soula 2002a, b) \\
\hline Chlorota aulica Burmeister, 1844 & $\begin{array}{l}\text { Argentina, Bolivia, Brazil (Alagoas to Pernambuco, Bahia, Espírito Santo, São Paulo), Colombia, Costa } \\
\text { Rica, Equador, Guatemala, French Guiana, Honduras, Mexico, Nicaragua, Panama, Peru, Suryname, } \\
\text { Venezuela (Soula, (Soula 2002a, b and present study) }\end{array}$ \\
\hline Chlorota espiritosantensis Ohaus, 1912 & Brazil (Bahia (new occurrence register), Espírito Santo) (Soula 2002a, b) \\
\hline Chlorota paulistana Ohaus, 1912 & Brazil (Bahia, São Paulo, Rio de Janeiro, Espírito Santo, Minas Gerais) (Soula 2002a and present study) \\
\hline \multicolumn{2}{|l|}{ Dorysthetus Blanchard, 1845} \\
\hline Dorysthetus espiritosantensis Ohaus, 1905 & Brazil (Bahia (new occurrence register), Espírito Santo) (Soula 2003) \\
\hline Dorysthetus fulgidus (Waterhouse, 1881) & $\begin{array}{l}\text { Brazil (Amazonas, Bahia (new occurrence register)), Colombia, Equador, Peru (Machatschke 1972, } \\
\text { Soula 1998, 2003) }\end{array}$ \\
\hline Dorysthetus taeniatus taeniatus (Perty, 1830) & Brazil (Bahia, Minas Gerais, Goiás) (Machatschke 1972, Soula 1998, 2003) \\
\hline \multicolumn{2}{|l|}{ Lagochile Hoffmannsegg, 1817} \\
\hline Lagochile amazona unipunctata (Ohaus, 1914) & Brazil (Bahia to Ceará) (Machatschke 1972, Soula 2005 and present study) \\
\hline Lagochile badia (Perty, 1830) & Brazil (Bahia, Goiás, Minas Gerais, São Paulo) (Machatschke 1972, Soula 2005) \\
\hline Lagochile bipunctata (MacLeay, 1819) & Argentina, Brazil (Bahia (Atlantic Forest), Espírito Santo, Rio de Janeiro) (Machatschke 1972, Soula 2005) \\
\hline Lagochile emarginata (Gyllenhal, 1817) & $\begin{array}{l}\text { Brazil (Bahia, Rio de Janeiro), occurs in mountains areas in the North at the Bahia state to Paraguay } \\
\text { Southwest, frequent in Brazilian Atlantic Forest to Argentina (Soula } 2005 \text { and present study) }\end{array}$ \\
\hline Lagochile emarginata nitida (Burmeister, 1844) & Brazil (Bahia) (Machatschke 1972, Soula 2005) \\
\hline Lagochile glandicolor (Burmeister, 1855) & Brazil (Amazonas, Bahia) (Soula 2005) \\
\hline Lagochile obscurata (Ohaus, 1905) & Brazil (Bahia), Colombia, French Guiana, Venezuela (Soula 2006) \\
\hline Lagochile obscurata debahia Soula, 2006 & Brazil (Bahia) (Soula 2006) \\
\hline Lagochile sparsa litoralis Ohaus, 1903 & Brazil (Bahia, Ceará, São Paulo) (Soula 2005 and present study) \\
\hline \multicolumn{2}{|l|}{ Macraspis MacLeay, 1819} \\
\hline Macraspis chrysis (Linnaeus, 1764) & $\begin{array}{l}\text { Brazil (Bahia to Santa Catarina), Colombia, Costa Rica, North of the Argentina to Mexico and, to } \\
\text { Paraguay, Peru to Bolivia, Nicaragua (Soula 1998) }\end{array}$ \\
\hline Macraspis cincta (Drury, 1782) & Brazil (Bahia (new occurrence register), Espírito Santo, Rio de Janeiro, São Paulo) (Soula 1998, 2003) \\
\hline Macraspis festiva Burmeister, 1844 & Bolivia, Brazil, Peruvian Amazon to Equador, and Paraguay, Venezuela (Soula 1998, Soula 2003) \\
\hline Macraspis morio Burmeister, 1844 & Argentina, Brazil, Colombia, Venezuela (Soula 2003 and present study) \\
\hline Macraspis nitidissima Burmeister, 1844 & Brazil (Bahia) (Soula 1998) \\
\hline Macraspis pseudochrysis pseudochrysis Landin, 1956 & Brazil (Bahia), French Guiana, Guyana, Peruvian Amazon and Venezuela (Soula 1998) \\
\hline Macraspis viridis (Thunberg, 1822) & Brazil (Bahia), Colombia (Machatschke 1972 and present study) \\
\hline \multicolumn{2}{|l|}{ Paramacraspis Ohaus, 1915} \\
\hline Paramacraspis hemichlora (Laporte, 1840) & Brazil (Bahia, Espírito Santo) (Soula 2002a, 2003 and present study) \\
\hline \multicolumn{2}{|c|}{ Pseudodorysthetus Soula, 1998 (new occurrence register) } \\
\hline Pseudodorysthetus calcaratus (Spínola, 1835) & Brazil (Bahia (new occurrence register), Rio de Janeiro) (Soula 1998, 2003) \\
\hline \multicolumn{2}{|l|}{ Areodina Burmeister, 1844} \\
\hline Areoda espiritosantensis Ohaus, 1905 & Brazil (Bahia (new occurrence register), Minas Gerais, Rio de Janeiro, São Paulo) (Ratcliffe \& Jameson 1989) \\
\hline Areoda leachii MacLeay, 1819 & Brazil (Bahia, Espírito Santo, Rio de Janeiro, São Paulo) (Ratcliffe \& Jameson 1989 and present study) \\
\hline
\end{tabular}


Table 1. Continued..

\section{SPECIES/SUBSPECIES}

Byrsopolis Burmeister, 1844 (new occurrence register)

B. laticollis Burmeister, 1855

Byrsopolis sp. nov.

Oplognathus MacLeay, 1819

Oplognathus bahianus Ohaus, 1912

Pelidnotina Burmeister, 1844

Chalcoplethis Burmeister, 1844

Chalcoplethis kirbii kirbii (Gray, 1832)

\section{DISTRIBUTION}

Brazil (Bahia (new occurrence register), Rio de Janeiro) (Machatschke 1972)

Brazil (Bahia)

Brazil (Bahia) (Machatschke 1972)

Brazil (Bahia, Espírito Santo, Paraná, Rio Grande do Sul) (Gray 1832, Burmeister 1844, Blanchard 1851, Harold 1869, Ohaus 1918, 1934, Blackwelder 1944, Machatschke 1972, Hardy 1975, Soula 2006, Krajcik 2008, Moore et al. 2017 and present study), Costa Rica (Hardy 1975, Moore et al. 2017), Paraguay (Cororó) (Moore et al. 2017)

Homonyx Guérin-Méneville, 1839

Homonyx bahianus Ohaus, 1913

Brazil (Bahia) (Ohaus 1913, 1918, 1934, Machatschke 1972, Krajcik 2008, Soula 2010, Moore et al. 2017 and present study)

Parhoplognathus Ohaus, 1915

Parhoplognathus rubripennis Ohaus, 1930

Brazil (Bahia) (Soula 2008, Moore et al. 2017)

Pelidnota MacLeay, 1819

Pelidnota alliacea (Germar, 1824)

Pelidnota bahiana bahiana Ohaus, 1905

Pelidnota beckeri Ferreira, Almeida \& Bravo, 2017

Pelidnota burmeisteri burmeisteri Burmeister, 1844

Pelidnota chalcothorax Perty, 1830

Pelidnota courtini Soula, 2009

Pelidnota crassipes Ohaus, 1905

Pelidnota cyanipes (Kirby, 1819)

Pelidnota cyanitarsis (Gory, 1833)

Pelidnota cuprea (Germar, 1824)

Pelidnota ebenina (Blanchard, 1842)

Pelidnota fulva Blanchard, 1851

Pelidnota glaberrima septentrionalis (Soula, 2006)

Pelidnota gracilis debahia (Soula, 2006)

Pelidnota instabilis Ohaus, 1912

Pelidnota lagoi Soula, 2011

Pelidnota liturella liturella (Kirby, 1818)

Pelidnota ludovici Ohaus, 1905

Brazil (Bahia, Espírito Santo, Santa Catarina) (Olivier 1789, 1802, Laporte 1840, Burmeister 1844 Blanchard 1851, Ohaus 1908, 1918, 1934, Blackwelder 1944, Machatschke 1972, Krajcik 2008, Soula 2009, Ferreira et al. 2017, Moore et al. 2017 and present study)

Brazil (Bahia) (Soula 2006, Moore et al. 2017)

Brazil (Bahia) (Ferreira et al. 2017 and present study)

Brazil (Bahia, Minas Gerais) (Machatschke 1972, Soula 2009, Moore et al. 2017 and present study)

Brazil (Bahia, Espírito Santo, Minas Gerais, Rio de Janeiro, São Paulo) (Perty 1830, Laporte 1840, Burmeister 1844, 1855, Blanchard 1851, Ohaus 1918a, 1918b, 1934, Blackwelder 1944, Machatschke 1972, Krajcik 2008, Soula 2009, Moore et al. 2017 and present study)

Brazil (Bahia, Minas Gerais) (Soula 2009, Moore et al. 2017 and present study)

Argentina (Misiones) (Ohaus 1905, 1918, 1934, Blackwelder 1944, Machatschke 1972, Soula 2006 , Krajcik 2008, Moore et al. 2017, Ferreira et al. 2017), Bolivia (Ohaus 1918, 1934, Blackwelder 1944, Moore et al. 2017, Ferreira et al. 2017), Brazil (Bahia, Minas Gerais, Goiás, Mato Grosso (Soula 2006, Moore et al. 2017, Ferreira et al. 2017 and present study), Paraguay (Asunción) (Ohaus 1905, 1918, 1934, Blackwelder 1944, Machatschke 1972, Soula 2006, Moore et al. 2017, Ferreira et al. 2017).

Argentina (Misiones) (Gutiérrez 1951, Soula 2009, Moore et al. 2017), Brazil (Pará, Bahia, Rio de Janeiro, Rio Grande do Sul) (Laporte 1840, Burmeister 1844, 1855, Blanchard 1851, Ohaus, 1908a, 1918b, 1934, Blackwelder 1944, Machatschke 1972, Krajcik 2008, Soula 2009, Moore et al. 2017 and present study)

Brazil (Bahia, Minas Gerais, Pará)) (Guérin-Méneville 1834, Burmeister 1844, Blanchard 1851, Ohaus 1918, 1934, Blackwelder 1944, Machatschke 1972, Krajcik 2008, Soula 2009, Moore et al. 2017 and present study)

Argentina (Soula 2006, Moore et al. 2017), Bolivia (Soula 2006, Moore et al. 2017), Brazil (Bahia, Goiás, Rio de Janeiro, Rio Grande do Sul, Santa Catarina) (Germar 1824, Perty 1830, Burmeister 1844, Blanchard 1851, Ohaus 1913, 1918, 1934, Blackwelder 1944, Machatschke 1972, Soula 2006, Krajcik 2008, Moore et al. 2017 and present study), Paraguay (Ohaus 1913, 1918, 1934, Machatschke 1972, Soula 2006, Moore et al. 2017)

Argentina (Soula 2006, Moore et al. 2017), Brazil (Pará, Bahia) (Ohaus 1908, 1918, 1934, Blackwelder 1944, Machatschke 1972, Soula 2006, Krajcik 2008, Moore et al. 2017 and present study), Bolivia (Santa Cruz) (Blanchard 1851, Ohaus 1918, 1934, Blackwelder 1944, Machatschke 1972, Soula 2006, Krajcik 2008, Moore et al. 2017)

Bolivia (Chuquisaca) (Blanchard 1851, Burmeister 1855, Ohaus 1918, 1934, Blackwelder 1944, Machatschke 1972, Krajcik 2008, Soula 2009, Moore et al. 2017), Brazil (Bahia, Minas Gerais, Mato Grosso do Sul) (Burmeister 1855, Ohaus 1908, Rodrigues \& da Silva Falco 2011, Rodrigues et al. 2012, Garcia et al. 2013, Moore et al. 2017)

Brazil (Bahia) (Soula 2006, Moore et al. 2017 and present study)

Brazil (Bahia) (Soula 2006, Moore et al. 2017 and present study)

Brazil (Bahia, Espírito Santo, Rio de Janeiro, São Paulo (Ohaus 1912, 1918, 1934, Blackwelder 1944, Machatschke 1972, Soula 2006, Krajcik 2008, Moore et al. 2017, Ferreira et al. 2017 and present study) Brazil (Bahia, Goiás) (Soula 2011, Moore et al. 2017, Ferreira et al. 2017 and present study) Argentina (Misiones) (Soula 2006, Moore et al. 2017), Brazil (Bahia, Goiás, Espírito Santo, Minas Gerais, Rio de Janeiro, Paraná, Santa Catarina, Rio Grande do Sul) (Burmeister 1844, 1855, Blanchard 1851, Ohaus 1908, 1918, 1929, 1934, Machatschke 1972, Soula 2006, Krajcik 2008, Moore et al. 2017 and present study)

Brazil (Bahia, Espírito Santo) (Machatschke 1972, Soula 2009, Moore et al. 2017 and present study). 
Table 1. Continued...

\section{SPECIES/SUBSPECIES}

Pelidnota pallidipennis F. Bates, 1904

Pelidnota rugulosa rugulosa Burmeister, 1844

Pelidnota semiaurata semiaurata Burmeister, 1844

Pelidnota sericeicollis (Frey, 1976)

Pelidnota sikorskii (Soula, 2006)

Pelidnota sordida (Germar, 1824)

Pelidnota sumptuosa (Vigors, 1825)

Pelidnota unicolor unicolor (Drury, 1778)

Pelidnota xanthospila (Germar, 1824)

Pelidnota sp. nov.

Rutelina MacLeay, 1819

Cnemida Kirby, 1827

Cnemida lacerata (Germar, 1824)

Cnemida retusa (Fabricius, 1801)

\section{DISTRIBUTION}

Brazil (Pernambuco, Bahia, Goiás, Minas Gerais, São Paulo, Mato Grosso) (Bates 1904, Ohaus 1918, 1934, Guimarães 1944, Blackwelder 1944, Machatschke 1972, Krajcik 2008, Soula 2009, Moore et al. 2017) Brazil (Bahia, Rio de Janeiro, São Paulo) (Burmeister 1844, 1855, Blanchard 1851, Ohaus 1918, 1934, Blackwelder 1944, Machatschke 1972, Soula 2006, Krajcik 2008, Moore et al. 2017, Ferreira et al. 2017 and present study)

Brazil (Bahia, Rio de Janeiro (INPA), Rio Grande do Sol, Santa Catarina (Ohaus 1918, 1934, Machatschke 1972, Krajcik 2008, Soula 2009, Moore et al. 2017, Ferreira et al. 2017 and present study) Brazil (Bahia (Encruzilhada)) (Frey 1976, Soula 2006, Krajcik 2008, Moore et al. 2017 and present study) Brazil (Bahia (Povoado de Cachimbo)) (Soula 2006, Moore et al. 2017 and present study) Argentina (Ohaus 1918, 1934, Blackwelder 1944, Machatschke 1972, Moore et al. 2017), Brazil (Bahia, Goiás, Minas Gerais, Rio de Janeiro, São Paulo, Paraná) (Burmeister 1844, 1855, Ohaus 1908, 1918a, 1934b, Guimarães 1944, Blackwelder 1944, Machatschke 1972, Krajcik 2008, Soula 2009, Moore et al. 2017 and present study), Paraguay (Ohaus 1918, 1934, Blackwelder 1944, Machatschke 1972, Moore et al. 2017)

Brazil (Pará, Bahia, Goiás, Minas Gerais, São Paulo, Mato Grosso) (Vigors 1825, Burmeister 1844, 1855, Blanchard 1851, Ohaus 1918, 1934, Blackwelder 1944, Machatschke 1972, Krajcik 2008, Soula 2009), Colombia (Caquetá, Meta) (Restrepo-Giraldo et al. 2003, Soula 2009, Pardo-Locarno et al. 2011), Paraguay (Soula 2009, Moore et al. 2017)

Brazil (Pernambuco, Bahia (new occurrence register), Espírito Santo, Minas Gerais, Rio de Janeiro, São Paulo, Santa Catarina) (Herbst 1790, Laporte 1840, Burmeister 1844, Blanchard 1851, Harold 1869, Ohaus 1908, 1913, 1918, 1934, Guimarães 1944, Machatschke 1972, Krajcik 2008, Soula 2009, Moore et al. 2017), Peru (Ratcliffe et al. 2015, Moore et al. 2017)

Brazil (Bahia, Espírito Santo, Minas Gerais, Rio de Janeiro, São Paulo, Santa Catarina) (Laporte 1840, Burmeister 1844, Blanchard 1851, Ohaus, 1918, 1934, Machatschke 1972, Soula 2006, Krajcik 2008, Moore et al. 2017 and present study)

Brazil (Bahia)

Brazil (Bahia, Distrito Federal (Brasília), Espirito Santo (Santa Leopoldina, Parati - Rolândia), Rio de Janeiro (Corcovado), Santa Catarina (Nova Teutônia - 271 1', 52023', Corupá, Joinville, Blumenau)) (Machatschke 1972, Jameson 1996)

Brazil (Bahia (Lençóis, Mucuri), Amapá (Porto Santana, Serro do Navio), Acre (Rio Humaits), Amazonas (Tefé, Manaus, Manacapurú, BR 319 km 275, Rio Javari, São Paulo do Olivença, Rio Tonantins, Rio Juruá, Fonte Boa), Espírito Santo (Linhares, Linhares, (P.N. Sooretama), Santa Leopoldina), Goiás (Jataí, Rio Verde, Trindade), Mato Grosso (Chapada dos Guimarães, Gleba Arinos, Reserva Humboldt ( $\left(10^{\circ} 11^{\prime} \mathrm{S}-59^{\circ} 48^{\prime} \mathrm{W}\right)$ ); Mato Grosso do Sul (Corumbá, Urucum), Minas Gerais, Pará, (Obidos, Obidos (Canta Galo), Colônia Rio Branco, Mocajuba, Est. Cruz Alta (Rio Trombetas), Santarém, Itaituba, Ilha de Marajó, Cameta, Mosquiero (Rio de Pará)), Amazonas (Faro); Rio de Janeiro (Jurujuba, Corcovado), Rondônia (Porto Velho (Rio Madeira), Ouro Preto do Oeste)), Bolivia (Beni, Villa Bella, Chuquisaca, El Palmar, Cochabamba, Rio Chapare), Colombia, Equador (Imbabura Pastaza, Rio Cururay), French Guiana (Cayenne, Cayenne, Roches de Kourou, Gourdonville, Charvein, Passoura (stream)), Guyana (West Berbice, Blairmont, Mazaruni-Potaro, Kartabu), Peru (Cuzco, Rio Vilcanota, Junin, 3-7 km SSW San Martin de Pangoa, Loreto, Ucayali R. Yarina Cocha, Rio Napo, Iquitos, Pucallpa (5 mi radius), Chambireyaci nr. Yurimaguas, Yurimaguas, San Martin, Mayobambo, Tarapoto), Suryname (Para, Dist. 13 Zanderij Area), Venezuela (Bolivar, Rio Caura, Distrito Federal, Caracas) (Jameson 1996 and present study)

\begin{tabular}{l} 
Rutela Latreille, 1802 \\
\hline Rutela histrio Sahlberg, 1823
\end{tabular}

Bolivia (Beni, Santa Cruz), Brazil (Amazonas, Bahia (Povoado de Cachimbo), Espírito Santo, Pará, Minas Gerais, Rio de Janeiro)), Colombia (Caqueta, Huila, Putumay), Equador (Loja, Morona-Santiago, Napo, Pastaza, Zamora, Chinchipe), French Guiana (Cayenne, Saint Laurent Du Moroni), Guyana (Mazaruni-Potaro), Paraguay, Peru (Amazonas, Junin, Hunaco, Lima, Loreto, Madre de Dios, Martin), Suryname, Venezuela (Bolivar, Monagas) (Jameson 1997 and present study)

ANATISTINI Lacordaire, 1856

Spodochlamys Burmeister, 1855

Spodochlamys caesarea Burmeister, 1855

Brazil (Bahia, Pará and São Paulo), French Guiana (Cayenne) and Trinidad (Machatschke 1972, Jameson \& Ratcliffe 2011, Ohaus 1918a and present study)

GENIATINI MacLeay, 1819

Bolax Fisher von Waldheim, 1829

Bolax audiberti Soula, 2011

Bolax flavolineata (Mannerheim, 1829)

Bolax sulcicollis (Germar, 1824)

Bolax sulcipennis Ohaus, 1928

Brazil (Bahia) (Soula 2011)

Brazil (Bahia (new occurrence register), Minas Gerais, Rio de Janeiro, São Paulo) (Jameson \& Hawkins 2005)

Brazil (Bahia, Espírito Santo) (Machatschke 1972, Jameson \& Hawkins 2005, Soula 2011)

Brazil (Bahia) (Machatschke 1972, Jameson \& Hawkins 2005, Soula 2011) 
Table 1. Continued...

\begin{tabular}{|c|c|}
\hline SPECIES/SUBSPECIES & DISTRIBUTION \\
\hline \multicolumn{2}{|l|}{ Geniates Kirby, 1819} \\
\hline Geniates immaculatus Camerano, 1878 & Brazil (Jameson \& Hawkins 2005 and present study) \\
\hline Geniates verticalis Burmeister, 1844 & Brazil (Jameson \& Hawkins 2005 and present study) \\
\hline \multicolumn{2}{|l|}{ Leucothyreus MacLeay, 1819} \\
\hline Leucothyreus acanthurus Ohaus, 1917 & Brazil (Bahia (new occurrence register) and Espírito Santo) (Jameson \& Hawkins 2005) \\
\hline Leucothyreus albopilosus Ohaus, 1917 & $\begin{array}{l}\text { Brazil (Acre, Bahia (new occurrence register), Espírito Santo, Mato Grosso, Rio de Janeiro, São Paulo) } \\
\text { (Jameson \& Hawkins 2005) }\end{array}$ \\
\hline Leucothyreus campestris Burmeister, 1855 & Brazil (Bahia (new occurrence register), Minas Gerais) (Jameson \& Hawkins 2005) \\
\hline Leucothyreus cayapo Ohaus, 1931 & Brazil (Bahia (new occurrence register), Goiás) (Jameson \& Hawkins 2005) \\
\hline Leucothyreus duplopunctatus Frey, 1976 & Brazil (Bahia (new occurrence register), Mato Grosso) (Jameson \& Hawkins 2005) \\
\hline Leucothyreus eligius Ohaus, 1918 & Brazil (Bahia (new occurrence register), Minas Gerais, Rio de Janeiro, São Paulo) (Jameson \& Hawkins 2005) \\
\hline Leucothyreus flavipes (Eschscholtz, 1822) & Brazil, Paraguay, Uruguay (Jameson \& Hawkins 2005 and present study) \\
\hline Leucothyreus fluminensis Ohaus, 1918 & Brazil (Bahia (new occurrence register) and Rio de Janeiro) (Machatschke 1972, Jameson \& Hawkins 2005) \\
\hline Leucothyreus garbei Ohaus, 1931 & Brazil (Bahia) (Machatschke 1972, Jameson \& Hawkins 2005 and present study) \\
\hline Leucothyreus iridipennis Ohaus, 1917 & Brazil (Bahia (new occurrence register), Espírito Santo, Santa Catarina) (Jameson \& Hawkins 2005) \\
\hline Leucothyreus kulzeri Frey, 1976 & Brazil (Bahia) (Jameson \& Hawkins 2005) \\
\hline Leucothyreus lucipetens Ohaus, 1931 & Brazil (Bahia (new occurrence register), Rio de Janeiro) (Jameson \& Hawkins 2005) \\
\hline Leucothyreus occipitalis Ohaus, 1931 & Brazil (Bahia (new occurrence register), Rio de Janeiro) (Jameson \& Hawkins 2005) \\
\hline Leucothyreus opacus (Perty, 1832) & Brazil (Jameson \& Hawkins 2005 and present study) \\
\hline Leucothyreus pallefactus Ohaus, 1924 & Brazil (Bahia (new occurrence register), Santa Catarina) (Jameson \& Hawkins 2005) \\
\hline Leucothyreus pallidus Ohaus, 1918 & Brazil (Bahia) (Jameson \& Hawkins 2005) \\
\hline Leucothyreus paulista Ohaus, 1917 & Brazil (Bahia (new occurrence register), São Paulo) (Jameson \& Hawkins 2005) \\
\hline Leucothyreus punctulatus Blanchard, 1851 & Brazil (Bahia (new occurrence register), Rio de Janeiro) (Jameson \& Hawkins 2005) \\
\hline Leucothyreus severinus Ohaus, 1918 & Brazil (Bahia) (Jameson \& Hawkins 2005) \\
\hline Leucothyreus similis Frey, 1976 & Brazil (Bahia) (Jameson \& Hawkins 2005 and present study) \\
\hline Leucothyreus spinifer Ohaus, 1918 & $\begin{array}{l}\text { Argentina (Salta), Brazil (Bahia, Minas Gerais, Rio de Janeiro), Paraguay (San Pedro) (Jameson \& } \\
\text { Hawkins } 2005 \text { and present study) }\end{array}$ \\
\hline Leucothyreus subcupreus Blanchard, 1851 & Brazil (Bahia) (Jameson \& Hawkins 2005) \\
\hline Leucothyreus suturalis Laporte, 1840 & Brazil (Bahia (new occurrence register), Espírito Santo, Santa Catarina) (Jameson \& Hawkins 2005) \\
\hline Leucothyreus trochantericus Ohaus, 1917 & Brazil (Bahia (new occurrence register), Rio de Janeiro) (Jameson \& Hawkins 2005) \\
\hline Leucothyreus verticalis Ohaus, 1924 & Brazil (Bahia (new occurrence register), Espírito Santo) (Jameson \& Hawkins 2005) \\
\hline Leucothyreus sp. 1 & Brazil (Bahia (Vitória da Conquista)) \\
\hline Leucothyreus sp. 2 & Brazil (Bahia (Mucugê)) \\
\hline Leucothyreus sp. 3 & Brazil (Bahia (Santa Terezinha)) \\
\hline Leucothyreus sp. 4 & Brazil (Bahia (Maracás)) \\
\hline Leucothyreus sp. 5 & Brazil (Bahia (Mucugê, Santa Terezinha, Salvador, Sauípe, Senhor do Bonfim)) \\
\hline Leucothyreus sp. 6 & Brazil (Bahia (Mucugê, Lençóis, Palmeiras)) \\
\hline Leucothyreus sp. 7 & Brazil (Bahia (Paulo Afonso)) \\
\hline Leucothyreus sp. 8 & $\begin{array}{l}\text { Brazil (Bahia (Feira de Santana, Maracás, Morro do Chapéu, Mucugê, Paulo Afonso, Santa Terezinha, } \\
\text { Sauípe, Vitória da Conquista)) }\end{array}$ \\
\hline Leucothyreus sp. 9 & Brazil (Bahia (Igrapiúna, Ituberá, Santa Terezinha, Porto Seguro)) \\
\hline Leucothyreus sp. 10 & Brazil (Bahia (Maracás)) \\
\hline \multicolumn{2}{|l|}{ Lobogeniates Ohaus, 1917} \\
\hline Lobogeniates alvinus Ohaus, 1931 & Brazil (Bahia) (Machatschke 1972, Jameson \& Hawkins 2005 and present study) \\
\hline Lobogeniates nigricans Ohaus, 1917 & Brazil (Bahia (Povoado Cachimbo)) (Jameson \& Hawkins 2005) \\
\hline Lobogeniates sp. 1 & Brazil (Bahia (Santa Terezinha)) \\
\hline Lobogeniates sp. 2 & Brazil (Bahia (Santa Terezinha)) \\
\hline Lobogeniates sp. 3 & Brazil (Bahia (Ituberá)) \\
\hline Lobogeniates sp. 4 & Brazil (Bahia (Ituberá)) \\
\hline \multicolumn{2}{|c|}{ Trizogeniates Ohaus 1917 (new occurrence register) } \\
\hline Trizogeniates planipennis Ohaus, 1917 & $\begin{array}{l}\text { Brazil (Bahia (new occurrence register), Espírito Santo, Minas Gerais, Rio Grande do Sul, Rio de } \\
\text { Janeiro, Santa Catarina, São Paulo), Peru (Jameson \& Hawkins 2005) }\end{array}$ \\
\hline
\end{tabular}


1912, Dorysthetus espiritosantensis Ohaus, 1905, D. fulgidus (Waterhouse, 1881), Leucothyreus acanthurus Ohaus, 1917, L. albopilosus Ohaus, 1917, L. campestris Burmeister, 1855, L. cayapo Ohaus, 1931, L. duplopunctatus Frey, 1976, L. eligius Ohaus, 1918, L. fluminensis Ohaus, 1918, L. iridipennis Ohaus, 1917, L. lucipetens Ohaus, 1931, L. occipitalis Ohaus, 1931, L. pallefactus Ohaus, 1924, L. paulista Ohaus, 1917, L. punctulatus Blanchard, 1851, L. suturalis Laporte, 1840, L. trochantericus Ohaus, 1917, L. verticalis Ohaus, 1924, Macraspis cincta (Drury, 1872), Paranomala tricostulata (Ohaus, 1897), P. violacea (Burmeister, 1844), Pseudodorysthetus calcaratus (Spinola, 1835), and Trizogeniates planipennis Ohaus, 1917. The subspecies Pelidnota unicolor unicolor (Drury, 1778) was also a new record for Bahia state (Table 1). Gathering this information with the already existing, makes a total of 101 species, and 17 subspecies in 4 tribes and 23 genera (Table 1) only for Bahia. Rutelini presented the greatest richness, with 16 genera, 49 species and 16 subspecies. Geniatini is the second tribe in richness with 5 genera and 46 species (Table 1). Anomalini and Anatistini presented only 1 genera each - Paranomala Casey, 1915, with 5 species and 2 subspecies and Spodochlamys Burmeister, 1855, with only one species (Table 1).

The richest genera were Leucothyreus (Geniatini), with 25 species, and Pelidnota (Rutelini) with 19 species and 9 subspecies (Figure 2). Leucothyreus, with 164 species described (Jameson \& Hawkins 2005), and Pelidnota, with 136 species and 60 valid subspecies (Moore et al. 2017, Ferreira et al. 2017), are the richest genera within Rutelinae.

There were recorded Ruteliane species in fifty-seven, taking into account the literature and the examined material, out of a total of 417 Bahian municipalities. Rutelini were found to occur in 41 localities, and Geniatini in 37 (Figure 1), which represents only $13.67 \%$ of the total amount of municipalities. Anomalini was recorded in only eight localities in the state and Anatistini only in the municipality of Cachoeira (Figure 1). Rutelini and Geniatini were recorded in all the Biomes, however Rutelini presented a concentration of records in areas of Atlantic Rain Forest near the coast. Geniatini had similar numbers of records in the Caatinga and in the Atlantic Forest (Figure 1). These findings could be attributed to the natural history of these tribes which immatures of Rutelini species occurs in dead wood
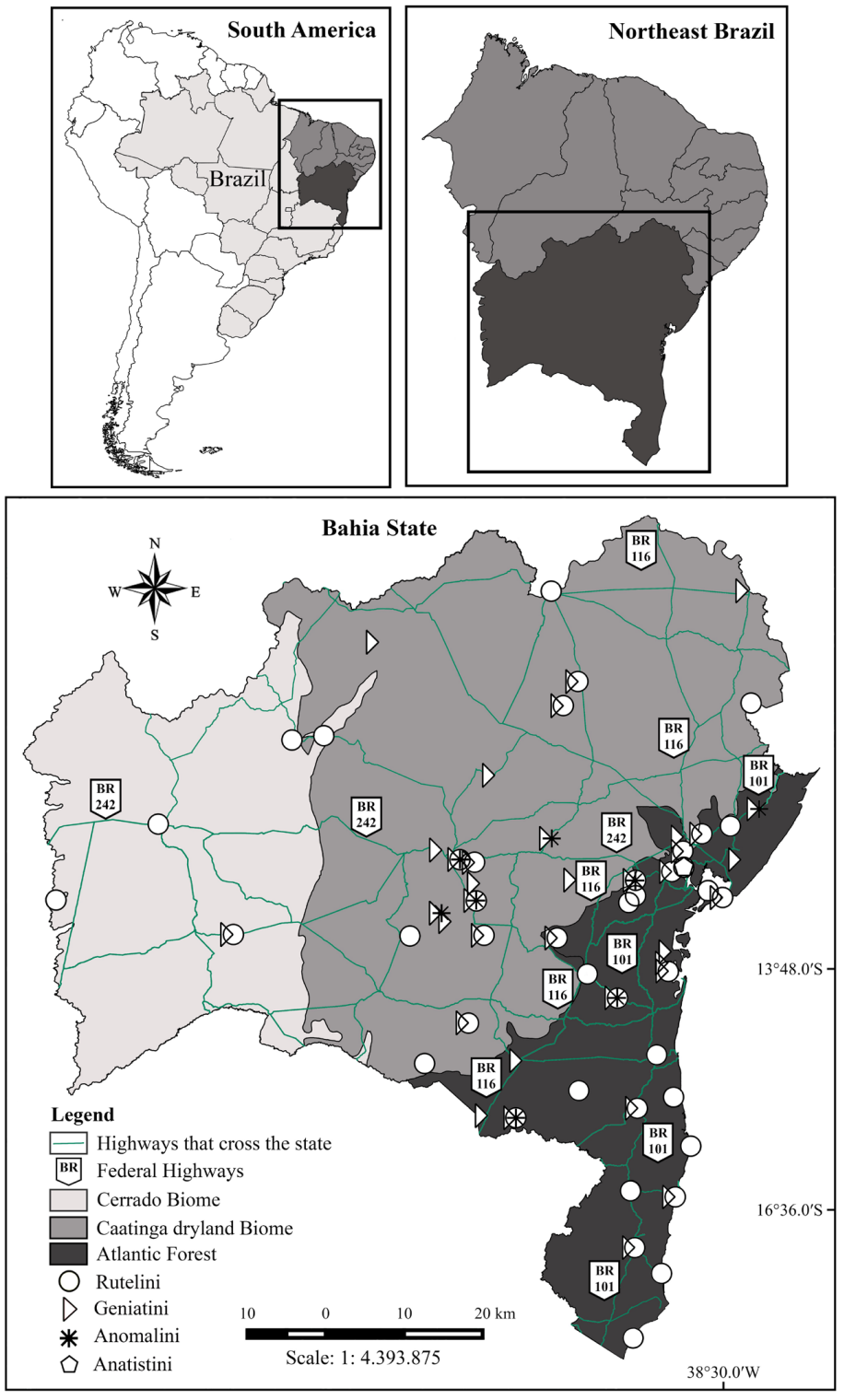

Figure 1. Location of the study area and geographic distribution records Rutelinae in the Bahia state. 


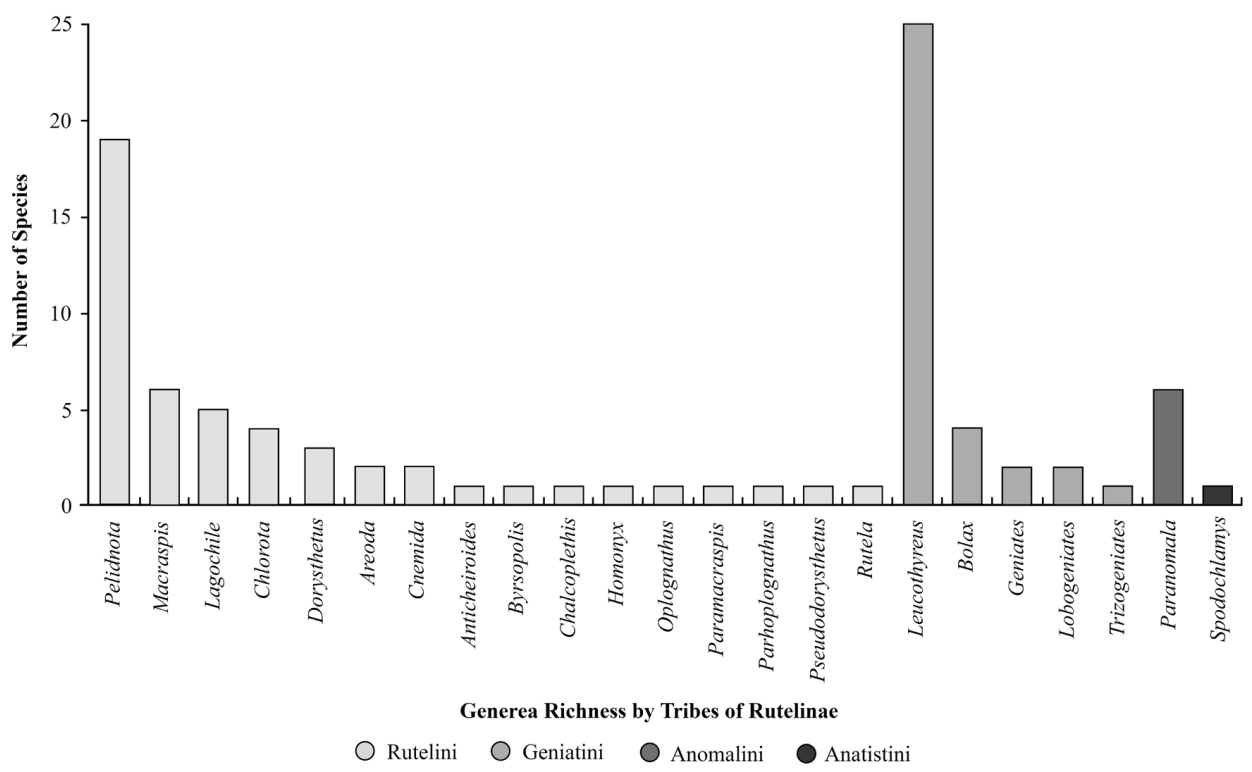

Figure 2. Species richness of Rutelinae from Bahia state, gathering the literature data with the results presented in this study.

(Albertoni et al. 2014), while Geniatini species develop mainly in the ground (Furhmann 2013).

The distribution of the Rutelinae species in Bahia has had, in part, direct correlation with the collection trips of the Projeto de Pesquisa em Biodiversidade do Semiárido (PPBio/Ministério da Ciência, Tecnologia, Inovações e Comunicações, MCTIC) since 2005. The ruteline specimens of PPBio Semiarido were deposited in the MZFS and these represent $72.8 \%$ (1,064 specimens) of all specimens examined in this study, with 68 species and 5 subspecies. However, although the PPBio has contributed significantly to expand the records of the Rutelinae from Bahia, there are still significant gaps to be explored in the state. The west, southwest and north regions of Bahia present the largest gaps (Figure 1). Certainly, there are many species to be discovered in these regions of the state, which demonstrates the need of a greater collection effort in the regions mentioned above (Oliveira et al. 2016). In addition to the mentioned issues, many of the subfamily distribution records in the state are located in areas near the highways that cross Bahia (Figure 1). Oliveira et al. (2016) report that species composition decreases as distance from access routes increases, and they suggest that collection localities distant from access routes could increase the possibility of new geographic records as well as records of new species. Most of the records for the south region of Bahia, in Belmonte, Barrolândia, Camacan, Itamaraju, Mucuri, Porto Seguro, Prado and Una, and two localities in the southern region: Encruzilhada and Cândido Sales, are the result of material deposited at MNRJ, CERPE and EPGC (Suplementary Material). Similarly, the collection points located in the north of the state are also from material deposited in MNRJ (examined material). The records for the southern region, Aracatu and Vitória da Conquista (examined material), are deposited in the MFS and were collected by the author.

Among the biomes present in Bahia, the Atlantic Forest has the highest representativity of Rutelinae records, with 32 localities of occurrence: Caatinga with 20 (with the largest collects gap in the southwest, and especially in the north of the state (Figure 1)) and the Cerrado, with only 5 points of records, representing the largest collects gap of all biomes (Figure 1). Atlantic Forest originally accounted for $1,300,000 \mathrm{~km}^{2}$ throughout 17 Brazilian states, with 120 million people living in this biome. However, only $22 \%$ of the original cover remains, and only $8.5 \%$ is in well preserved protection areas, on fragments of over 100 hectares (MMA 2017). Caatinga covers $844,453 \mathrm{~km}^{2}$, representing $11 \%$ of the national territory, and is present in nine states of Northeastern Brazil and in the north of Minas Gerais state (MMA 2017). The Caatinga and the Cerrado has been deforested in an accelerated way, especially in the last years, and $46 \%$ of the Caatinga total original cover has been deforested (Flores et al. 2012, MMA 2017). Cerrado has been historically transformed in extensive crop plantations in Brazil, causing an increasing reduction of the vegetal cover due to the progressive increase in agricultural economic activity in areas in its domain (Flores et al. 2012). This reduction in vegetal cover has, in turn, generated direct consequences for biodiversity loss (Queiroz 2009, MMA 2017). However, only $7.6 \%$ of the total area of the Caatinga is in the protection area, and only $1 \%$ of this area is designated as Units of Integral Protection (MMA 2017). In addition, approximately $30 \%$ of the Caatinga has already been altered due to human action, and especially due to agriculture. In view of these factors, there is a risk of the rapid loss of unique species in this biome (Araújo et al. 2005), many of which are yet unknown to science. The Cerrado biome presents the largest collects gap of Rutelinae in Bahia state. Cerrado covers 2,036,448 $\mathrm{km}^{2}$ (representing $22 \%$ of the national territory), and is present in 12 Brazilian states. It is considered a hotspot of world biodiversity, with an extreme abundance of endemic species (MMA 2017). However, the Cerrado is the biome with the lowest percentage of areas of integral protection. Only $8.21 \%$ of the total territory is legally protected by Units of Conservation; of this total, $2.85 \%$ are Units of Conservation of Integral Protection and 5.36\% are Units of Conservation of Sustainable Use, including Particular Reserve of Natural Heritage - RPPNs (0.07\%) (MMA 2017). The findings presented above demonstrate the need to make more collects in the state, especially in Cerrado in the west region of the state, to enable a better understanding of the distribution, expanding the knowledge of the group.

With the results presented here there was an expressive increase, both in the richness of genera and species. After this study the number of genera increased to 23, with the new records of Byrsopolis Burmeister, 
Pseudodorysthetus Soula and Trizogeniates Ohaus; new distributional records of 30 species and 1 subspecies from Bahia state, and with 14 species, that will be described in subsequent papers, 10 of Leucothyreus (according to the consultation with a researcher, Seidel M., who is conducting the review of the genus), 2 of Lobogeniates (a genus in process of review by ASF) and 1 of Byrsopolis and Pelidnota. With respect to the number of registered species of Rutelinae in Bahia, was increased in more than a half, reaching 101 species and 17 subspecies.

This increase in the number of registered Rutelinae species demonstrates the importance of conducting inventories for group knowledge. In addition, the distribution of the Rutelinae locality records in the Bahia state in only 57 registered municipalities, which is equivalent to $13.67 \%$ of the total municipalities in the state, plus the gaps seen in the most richest and poorly known biomes, emphasizes the imminent need for new studies involving the Rutelinae for a better knowledge of fauna, not only in the biomes of this state, but in Brazil as a whole.

\section{Supplementary material}

The following online material is available for this article:

Supplementary material

\section{Acknowledgments}

The authors would like to thank to the Coordenação de Aperfeiçoamento de Pessoal de Nível Superior (CAPES) for the master's fellowship to the first author (ASF), Conselho Nacional de Desenvolvimento Científico e Tecnológico (CNPq) for the research fellowship awarded to LMA (306772/2006-0) and FB (305055/2012-7), Dra. Marcela Monné and Dr. Miguel A. Monné (MNRJ), Dra. Jane Costa and Dr. Márcio Félix (CEIOC-RJ), Dr. Everardo J. Grossi (EPGC), and Dra. Favízia F. Oliveira (MHNBA/MZUFBA) for allowing the study of material, the PPBio Semiarido project (Programa de Pesquisa em Biodiversidade do Semiárido) (agreement CNPq/MCTI - Ministério da Ciência, Tecnologia e Inovação n.o: 457471/2012-3) for financial support for collections and for supporting to visit of Paschoal Grossi visit to the collections at MZFS.

\section{Authors Contributions}

André da Silva Ferreira: Contribution to data collection, contribution to data analysis and interpretation, contribution to manuscript preparation and contribution to critical revision, adding intellectual content.

Lúcia M. Almeida and Freddy Bravo: Contribution to critical revision, adding intellectual content.

Paschoal Coelho Grossi: Contribution to data collection, critical revision, adding intellectual content.

\section{Conflicts of interest}

The authors declare that they have no conflict of interest related to the publication of this manuscript.

\section{References}

ALBERTONI, F.F., FUHRMANN, J. \& IDE, S. 2014. Lagochile emarginata (Gyllenhal): morphology of immature and imago, and biological records (Coleoptera, Scarabaeidae, Rutelinae). Revista Brasileira de Entomologia 58(1): 32-46.

ARAÚJO, F.S.; RODAL, M.J.N. \& BARBOSA, M.R.V. 2005. Análise das variações da biodiversidade do bioma Caatinga: suporte a estratégias regionais de conservação. Ministério do Meio Ambiente, DF. Brasília.
BAHIA. 2013. Meio Ambiente na Bahia. Secretaria de Indústria, Comércio e Mineração/Companhia Baiana de Pesquisa Mineral. http://www.cbpm.com. br/paginas/meio_bahia.php (last access at 14/09/2015).

BATES, F. 1904. A revision of the sub-family Pelidnotinae of the coleopterous family Rutelidae, with descriptions of new genera and species. Transactions of the Entomological Society of London 1904: 249-276.

BLANCHARD, C.E. 1850 (1851). Ordre des Coleoptera. In Muséum d'Histoire Naturelle de Paris. Catalogue de la Collection Entomologique. Classe des Insectes, Vol. 1, Part 2. (H. Milne-Edwards, C.E. Blanchard \& H. Lucas. eds.). Gide and Baudry, Paris, p.129-240.

BLACKWELDER, R.E. 1944. Checklist of the Coleopterous Insects of Mexico, Central America, the West Indies, and South America. United States National Museum Bulletin 185: 189-265.

BOUCHARD, P., BOUSQUET, Y., DAVIES, A. E., ALONSO-ZARAZAGA, M. A., LAWRENCE, J. F., LYAL, C. H., NEWTON, A. F., REID, C. A., SCHMITT, M., SLIPIŃSKI, S. A. \& SMITH, A. B. 2011. Family-group names in Coleoptera (Insecta). ZooKeys 88: 1-972.

BURMEISTER, H. 1844. Handbuch der Entomologie. (Coleoptera Lamellicornia Anthobia et Phyllophaga Systellochela), Vol. 4, Pt. 1. Berlin.

BURMEISTER, H. 1855. Handbuch der Entomologie. (Coleoptera Lamellicornia Anthobia et Phyllophaga Systellochela), Vol. 4., Pt. 2. Berlin.

CHERMAN, M.A. \& MORÓN, M.A. 2014. Validación de la Família Melolonthidae Leach, 1819 (Coleoptera: Scarabaeoidea). Acta Zoológica Mexicana (n.s.) 30(1): 201-220.

ENDRÖDI, S. 1966. Monographie der Dynastinae I Teil 1 Tribus Cyclocephalini. Entomologische Abhandlungen Staatlichen Museum für Tierkunde, Dresden 33: $1-145$.

EVANS, A.V. 2002. Melolonthinae. In American Beetles Volume 2. Polyphaga: Scarabaeoidea through Curculionoidea. (R.H. Arnett, M.C. Thomas, P.E. Skelley \& J.H. Frank. eds.). CRC Press, Boca Raton, Florida, p.51-60.

FABRICIUS, J.C. 1801. Systema Eleutheratorum Secundum Ordines, Genera, Species: Adiectis Synonymis, Locis, Observationibus, Descriptionibus, volume 2. Impensis Bibliopolii Academici Novi. Kiel, Germany.

FERREIRA, A.S., ALMEIDA, L.M. \& BRAVO, F. 2017. Three new species of Pelidnota MacLeay (Coleoptera, Scarabaeidae, Rutelinae) and new distributional records from northeast Brazil. Revista Brasileira de Entomologia 61: 208-223. http://doi.org/10.1016/j.rbe.2017.04.004.

FILIPPINI, V., MICÓ, E. \& GALANTE, E. 2016. Checklist and identification key of Anomalini (Coleoptera, Scarabaeidae, Rutelinae) of Costa Rica. ZooKeys 621: 63-136. http://doi:10.3897/zookeys.621.7565.

FLORES, M.P., GUIMARÃES, R.F., CARVALHO JÚNIOR, O.A. \& GOMES, R.A.T. 2012. Multitemporal Analysis of Agricultural Expansion in the Barreiras Municipality - Bahia. Campo-Território: revista de geografia agrária 7(14): 1-19.

FREY, G. 1976. Neue Sudamerikanischen Ruteliden. Entomologische Arbeiten aus dem Museum 27: 344-356.

FUHRMANN, J. 2013. Description of the third larval instar and pupa of Geniates barbatus Kirby (Coleoptera, Scarabaeidae, Rutelinae). Revista Brasileira de Entomologia 57(1): 40-46.

GARCIA, F.P., RODRIGUES, S.R., BAGNARA, C.A.C., DE OLIVEIRA, D.S. 2013. Survey of saproxylophagous Melolonthidae (Coleoptera) and some biological aspects in Aquidauana, MS. Biota Neotropica 13 (3): 38-43.

GERMAR, E.F. 1824. Insectorum species novae aut minus cognitae, descriptionibus illustratae. Halle.

GORY, H.L. 1833. Description de deux Coléoptères nouveaux des genres Rutela et Buprestis. Annales de la Société entomologique de France 2: 67-68.

GRAY, G. R. 1832. Supplement on the Lamellicornes. In The Class Insecta Arranged by the Baron Cuvier, with Supplementary Additions to each Order, Volume 1. Whittaker, Treacher, and Co. (E. Griffith \& E. Pidgeo. eds.). London, UK, p.504-537.

GROSSI, P.C. \& VAZ-DE-MELLO, F.Z. 2016. Rutelinae in Catálogo Taxonômico da Fauna do Brasil. PNUD. http://fauna.jbrj.gov.br/fauna/faunadobrasil/126897 (last access at 10/09/2016).

GUÉRIN-MÉNEVILLE, F.É. 1834. Iconographie du Règne Animal de G. Cuvier, ou Représentation d'Après Nature de L'une des Espèces les Plus Remarquables, 
et Souvent non Encore Figurées, de Chaque Genre d'Animaux. Avec un texte descriptif mis au courant de la science. Ouvrage Pouvant Servir d'Atlas à Tous les Traités de Zoologie. Insectes. Vol. 7. J. B. Baillière, Paris, 576 pp. [+ 110 plates].

GUIMARÃES, L.R. 1944. Rutelidae, Cetonidae, Melolonthidae e Dynastidae de Monte Alegre. Papéis Avulsos do Departamento de Zoologia (São Paulo) 6: 93-102.

GUTIÉRREZ, A.R. 1951. Notas sobre Scarabaeidae neotrópicos II (Coleopt. Lamellic.). Anales de la Sociedad Científica Argentina 151: 106-125.

HARDY, A.R. 1975. A revision of the genus Pelidnota of America north of Panama (Coleoptera: Scarabaeidae: Rutelinae). University of California Publications in Entomology 78: 1-43.

HARDY,A. 1991. A Catalog of the Coleoptera of America North of Mexico - Family: Scarabaeidae, Subfamilies: Rutelinae and Dynastinae. California, United States - Department of Agriculture, Agriculture Handbook.

HAROLD, E. 1869. Scarabaeidae. In Catalogus Coleopterorum Hucusque Descriptorum Synonymicus et Systematicus, Vol. 4. E. H. Gummi (M. Gemminger \& E. Harold. eds.). Munich, p.979-1346.

HAWKS, D.C. 2017. Five new species of Chrysina Kirby (Coleoptera: Scarabaeidae: Rutelinae). Insecta Mundi 0544: 1-9.

HERBST, J.F.W. 1790. Natursystem aller bekannten in- und ausländlischen Insekten, als eine Fortsetzung der von Büffonschen Naturgeschichte. Der Käfer dritter Theil. Pauli, Berlin.

HUNT, T., BERGSTEN, J., LEVKANICOVA, Z., PAPADOPOULOU, A, ST. JOHN, O., WILD, R., HAMMOND, P.M., AHRENS, D., BALKE, M., CATERINO, M.S., GÓMEZ-ZURITA, J., RIBERA, I., BARRACLOUGH, T.G., BOCAKOVA, M., BOCAK, L. \& VOGLER, A.P. 2007. A Comprehensive Phylogeny of of Beetles Reveals the Evolutionary Origins of a Superradiation. Science 318: 1913-1919.

IBGE-INSTITUTO BRASILEIRO DE GEOGRAFIA E ESTATÍSTICA. 2013. Área Territorial Brasileira: Consulta por Unidade de Federação. http://www.ibge.gov. br/home/geociencias/areaterritorial/principal.shtm (las access at 14/09/2015).

JAMESON, M.L. 1996. Revision and Phylogeny of the Neotropical genus Cnemida (Coleoptera: Scarabaeidae: Rutelinae). Insecta Mundi 10(1-4): 285-315.

JAMESON, M. L. 2002. Rutelinae MacLeay 1819. In American Beetles Volume 2. Polyphaga: Scarabaeoidea through Curculionoidea. (R.H. Arnett, M.C. Thomas, P.E. Skelley \& J.H. Frank eds.). CRC Press, Boca Raton, Florida, p.60-64.

JAMESON, M.L. 2008. Review of the genus Microchilus Blanchard (Coleoptera: Scarabaeidae: Rutelinae: Geniatini). Insecta Mundi 0025: 1-14.

JAMESON, M.L. \& HAWKINS, S.J. 2005. Synopsis of the genera of Geniatini (Coleoptera: Scarabaeidae: Rutelinae) with an annotated catalog of species. Zootaxa 874: 1-76.

JAMESON, M.L. \& RATCLIFFE, B.C. 2011. The Neotropical Scarab Beetle Tribe Anatistini (Coleoptera: Scarabaeidae: Rutelinae). Bulletin of the University of Nebraska State Museum 26: 1-100.

KOHLMANN, B. \& MORÓN, M. A. 2003. Análisis histórico de la clasificación de los Coleoptera Scarabaeoidea o Lamellicornia. Acta Zoológica Mexicana (n.s.) 90: 175-280

KRAJCIK, M. 2008. Checklist of Scarabaeoidea of the World: Rutelinae (Coleoptera: Scarabaeidae: Rutelinae), Vol. 2. Animma. X Supplement 4, Plzen Milan Krajcik.

LAPORTE [=CASTELNAU] FLNCde. 1840. Histoire naturelle des insectes Coléoptères; avec une introduction renfermant l'anatomie et la physiologie des animaux articulés, par M. Brullé. Tome premier. Histoire naturelle des animaux articulés, annélides, crustacés, arachnides, myriapodes et insectes Tome troisième. P. Duménil, Paris, 324p. [+ 19 plates].

LAWRENCE, J.F. \& NEWTON, A.F. 1995. Families and subfamilies of Coleoptera (with selected genera, notes and references, and data on family-group names) In Biology, Phylogeny, and Classification of Coleoptera: Papers Celebrating the 80th Birthday of Roy A. (J. Pakaluk \& S.A. Slipinski. eds.). Crowson, Warsaw: Muzeum i Instytut Zoologii PAN, p.779-1006.

LAWRENCE, J.F., ŚLIPIŃSKI, A., SEAGO, A. E., THAYER, M.K., NEWTON, A.F. \& MARVALDI, A.E. 2011. Phylogeny of the Coleoptera Based on Morphological Characters of Adults and Larvae. Annales Zoologici 61: 1-217.

MACHATSCHKE, J. 1972. Scarabaeidae: Melolonthidae, Rutelinae. Coleopterum Catalogus Supplementa 66(1-2): 1-429.
MMA-MINISTERIO DO MEIO AMBIENTE. 2017. Mata Atlântica. http://www. mma.gov.br/biomas/mata-atlantica (last access at 06/06/2017).

MOORE, M.R., JAMESON, M.L., GARNER, B.H., AUDIBERT, C., SMITH, A.B.T. \& SEIDEL, M. 2017. Synopsis of the pelidnotine scarabs (Coleoptera, Scarabaeidae, Rutelinae, Rutelini) and annotated catalog of the species and subspecies. ZooKeys 666: 1-349.

MORÓN, M.A. 1990. The Beetle of the World: Rutelini. Sciences Nat. 10: 1-206.

MORÓN, M. A. 2004. Melolontídeos edafícolas. In Pragas de solo no Brasil. (J.R. Salvadori, C.J. Ávila \& M.T.B. Silva. eds.). Passo Fundo: Embrapa Trigo; Dourados: Embrapa Agropecuária Oeste; Cruz Alta: Fundacep Fecotrigo, p.41-68.

MORÓN, M.A. \& RAMÍREZ-PONCE, A. 2012. Mesoamerican genera of Anomalin (Coleoptera: Melolonthidae: Rutelinae): A brief review. Trends in Entomology 8: 97-114.

MORÓN, M.A., RATCLIFFE, B.C. \& DELOYA, C. 1997. Atlas de los Escarabajos de México. Coleoptera: Lamellicornia. Vol. 1: Família Melolonthidae. Subfamílias Rutelinae, Dynastinae, Cetoniinae, Trichiinae, Valginae y Melolonthinae. Sociedad Mexicana de Entomologia, A.C., Mexico.

OHAUS, F. 1897. Stettiner Entomologische Zeitung, LVIII

OHAUS, F. 1905. Revision der Amerikanischen Anoplognathiden (Coleoptera Lamellicornia). Stettiner Entomologische Zeitung 1905: 120-167.

OHAUS, F. 1908. Die Ruteliden meiner Sammelreisen in Südamerika (Col.). Deutsche Entomologische Zeitschrift 1908: 239-262.

OHAUS, F. 1912. Beiträge zur Kenntnis der Ruteliden. X. Stettiner Entomologische Zeitung 73: 273-319.

OHAUS, F. 1913. XI. Beitrag zur Kenntnis der Ruteliden. (Col.). Deutsche Entomologische Zeitschrift 1913: 487-511.

OHAUS, F. 1917. Neue Geniatinen (Col. lamell. Rutelin.). Stettiner Entomologische Zeitung 78: 3-53.

OHAUS, F. 1918a. Scarabaeidae: Euchirinae, Phaenomerinae, Rutelinae. Coleopterum Catalogus 20: 1-124.

OHAUS, F. 1918b. Neue Geniatinen II (Col. lamell. Rutelin.). Stettiner Entomologische Zeitung 79: 350-365.

OHAUS, F. 1924. Neue Geniatinen III. Stettiner Entomologische Zeitung 84: 179-186.

OHAUS, F. 1926. Three new species of Rutelinae (Coleoptera lamellicornia) in the Carnegie Museum. Annals of the Carnegie Museum 17: 87-89.

OHAUS, F. 1928. Beitrag zur Kenntnis der Ruteliden (Col. lamell.). Deutsche Entomologische Zeitschrift 1928(1929): 385-406.

OHAUS, F. 1930. XXVI. Beitrag zur Kenntnis der Rutelinen (Col. amell.). Deutsche Entomologische Zeitschrift 1930: 138-158.

OHAUS, F. 1931. Neue Geniatinen IV. Stettiner Entomologische Zeitung. Stettin 92: $227-258$

OLIVEIRA, U., PAGLIA, A.P., BRESCOVIT, A.D., CARVALHO, C.J.B., SILVA, D.P., REZENDE, D.T., LEITE, F.S.F., BATISTA, J.A.N., BARBOSA, J.P.P.P., STEHMANN, J.R., ASCHER, J.S., VASCONCELOS, M.R., MARCO JR., P., LÖWENBERG-NETO, PP., DIAS, P.G., FERRO, V.G. \& SANTOS, A.J. 2016. The strong influence of collection bias on biodiversity knowledge shortfalls of Brazilian terrestrial biodiversity. Diversity and Distributions 2016: 1-13.

OLIVIER, A.G. 1789. Entomologie, ou Histoire Naturelle des Insectes, avec leurs Caractères Génériques et Spécifiques, leur Description, leur Synonymie, et leur Figure Enluminée. Coléoptères, Tome Premier (genera separately paged). Baudouin, Paris, 432p. [xix +2 pp]

OLIVIER, A.G. 1802. Entomologie oder Naturgeschichte der Insekten mit ihren Gattungs- und Art-Mekmalen, ihrer Beschreibung und Synonymie. Käfer Zweiter Theil. Mit Kupfern. Karl Reichard, Braunschweig.

PARDO-LOCARNO, L.C., RAMÍREZ-PAVA, B., VILLOTA, H., VILLANUEVA, O, \& BAHAMÓN, W. 2011. Ensamblaje de escarabajos Melolonthidae (Coleoptera Scarabaeoidea) asociados con pasturas en el departamento del Caquetá y su posible relación con la salubridad edáfica. Acta Agronómica 60 (3): 1-12.

PAUCAR-CABRERA, A. 2003. Systematics and phylogeny of the genus Epectinaspis Blanchard (Coleoptera: Scarabaeidae: Rutelinae) and description of a new genus of Anomalini from Mexico. Coleopterists Society Monographs 2: 1-60.

PEREIRA, A.F., RODRIGUES, S.R. \& MORÓN, M.A. 2013. Biological aspects of Leucothyreus alvarengai Frey and Leucothyreus aff. semipruinosus Ohaus 
(Coleoptera, Melolonthidae, Rutelinae) in crop succession at central Brazil. Revista Brasileira de Entomologia 57 (3): 323-228.

PERTY, J.A.M. 1830. Insecta Brasiliensia. In Delectus animalium articulatorum, quae in itinere per Brasilliam annis MDCCCXVII-MDCCCXX jussu et auspiciis Maximilliani Josephi I. Bavariae regis augustissimi peracto. (J. Spix \& C. Martius. eds.). Frid. Fleicher, Monachii [München], p.1-60.

PUKER, A., RODRIGUES, S.R., TIAGO, E.F. \& SANTOS, W.T. 2009. Phytophagous species of Scarabaeidae (Insecta: Coleoptera) associated with the root system of Acrocomia aculeata (Jacq.) Loddi. ex Mart. (Arecaceae). Biota Neotropica 9 (3): 105-109.

QUEIROZ, F.A. 2009. Impacts of the international trade of soybeans on the biodiversity of Cerrado Savannah. Sociedade \& Natureza, Uberlândia 21 (2): 193-209.

RATCLIFFE, B.C. \& JAMESON, M.L. 1989. A Synopsis of the Genus Areoda (Coleoptera: Scarabaeidae: Rutelinae). The Coleopterists Bulletin 43(2): 135-144.

RATCLIFFE, B.C., JAMESON, M.L., FIGUEROA, L., CAVE, R.D., PAULSEN, M.J., CANO, E.B., BEZA-BEZA, C., JIMENEZ-FERBANS. L. \& REYESCASTILLO, R. 2015. Beetles (Coleoptera) of Peru: A survey of families. Scarabaeoidea. The Journal of the Kansas Entomological Society 88: 186-207.

RESTREPO-GIRALDO, H., MORÓN, M.A., VALLEJO, F., PARDO-LOCARNO, L.C. \& LÓPEZ-AVILA, A. 2003. Catálogo de Coleoptera Melolonthidae (Scarabaeidae Pleurosticti) de Colombia. Folia Entomológica Mexicana 42: 239-263.

RODRIGUES, S.R., PUKER, A., ABOT, A.R.; BARBOSA, C.L., IDE, S. \& COUTINHO, G.V. 2008. Ocorrência e aspectos biológicos de Anomala testaceipennis Blanchard (Coleoptera, Scarabaeidae). Revista Brasileira de Entomologia 52 (1): 68-71.

RODRIGUES, S.R. \& DA SILVA FALCO, J. 2011. Aspectos biológicos de Pelidnota fulva Blanchard, 1850 (Coleoptera, Scarabaeidae, Rutelinae). Biota Neotropica 11: $157-160$.

RODRIGUES, S.R., MORÓN, M.A., NOGUEIRA, G.A.L. 2012. Description of the third instar of Pelidnota fulva Blanchard, 1850 (Coleoptera: Scarabaeidae: Rutelinae). The Coleopterists Bulletin 66 (3): 266-270.

RODRIGUEZ-DEL-BOSQUE, L.A. 1996. Population and adult longevity the Phyllophaga crinita, Anomala flavipennis and A. forminosa Coleoptera (Scarabaeidae). Southwestern Entomologist 21 (1): 55-58.

RODRIGUEZ-DEL-BOSQUE, L.A. 1998. A sixteen-year study on the bivoltinism of Anomala flavipennis (Coleoptera: Scarabaeidae) in Mexico. Environmental Entomology 27: 248-252

SEIDEL, M., JAMESON, M.L. \& STONE, R.L. 2017. A new cryptic species and review of the east-Andean leaf chafer genus Mesomerodon Ohaus, 1905
(Coleoptera, Scarabaeidae, Rutelinae). ZooKeys 671: 61-85. http://doi. org/10.3897/zookeys.671.11815.

SIERRA, J.M. 2017. Four new species of Chrysina Kirby (Coleoptera: Scarabaeidae: Rutelinae) from Guatemala and Honduras. Insecta Mundi 0543: 1-12.

SMITH, A.B.T., HAWKS, D.C. \& HERATY, J.M. 2006. An Overview of the Classification and Evolution of the Major Scarab Beetle Clades (Coleoptera: Scarabaeoidea) Based on Preliminary Molecular Analyses. Coleopterists Society Monograph 5: 35-46.

SOULA, M. 1998. Les Coléoptères du Nouveau Monde. Rutelini 2. Revision Des Anticheirina 1. Hillside Books, Canterbury 26 (1): 1-116.

SOULA, M. 2002a. Les Coléoptères du Nouveau Monde. Rutelini 2. Hillside Books, Canterbury 26: 1-98.

SOULA, M. 2002b. Les Coléoptères du Nouveau Monde. Rutelini 2. Hillside Books, Canterbury 26 (2): 117-296.

SOULA, M. 2003. Les Coléoptères du Nouveau Monde. Rutelini 3. Hillside Books, Canterbury 29: 1-76.

SOULA, M. 2005. Les Coléoptères du Nouveau Monde. Rutelini 3. Révision Des Anticheirina 3. Hillside Books, Canterbury 26 (3): 294-409.

SOULA, M. 2006. Les Coléoptères du Nouveau Monde. Rutelini 1. Révision des Pelidnotina 1 Et des Lasiocalina. Besoiro Suplement 1: 1-176.

SOULA, M. 2008. Les Coléoptères du Nouveau Monde. Rutelini 2. Révision des Pelidnotina 2, Besoiro, 2: 1-40.

SOULA, M. 2009. Les Coléoptères du Nouveau Monde. Rutelini 3. Révision des Pelidnotina 3, Besoiro 3: 1-139.

SOULA, M. 2010. Les Coléoptères du Nouveau Monde. Rutelini 4. Révision des Pelidnotina 4, Besoiro 4: 1-66.

SOULA, M. 2011. Les Coléoptères du Nouveau Monde. Rutelini 5. Geniatini 1, Révision du genre Bolax, Besoiro 5: 1-85.

VIANA, C.H.P., MARQUES, O.M. \& CARVALHO, C.A.L. 2001. Rutelinae (Coleoptera: Scarabaeidae) Coletados em Armadilha Luminosa em Cruz das Almas, Bahia. Magistra, Cruz Das Almas-Bahia 13 (1): 1-7.

VIGORS, N.A. 1825. Descriptions of some rare, interesting, or hitherto uncharacterized subjects of zoology. The Zoological Journal 1: 526-542.

Received: $30 / 10 / 2017$

Revised: 29/01/2018

Accepted: $31 / 01 / 2018$

Published online: 05/03/2018 